\title{
Decreased expression of dual-specificity phosphatase 9 is associated with poor prognosis in clear cell renal cell carcinoma
}

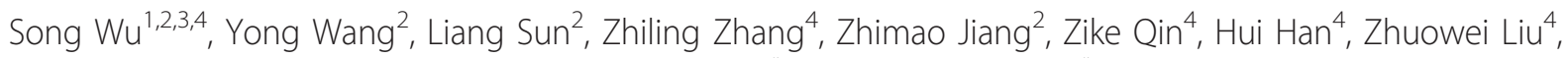
Xianxin $\mathrm{Li}^{2}$, Aifa Tang ${ }^{1}$, Yaoting Gui ${ }^{2}$, Zhiming Cai ${ }^{1,2,3^{*}}$ and Fangjian Zhou ${ }^{4^{*}}$

\begin{abstract}
Background: The molecular mechanisms involved in the development and progression of clear cell renal cell carcinomas (ccRCCs) are poorly understood. The objective of this study was to analyze the expression of dualspecificity phosphatase 9 (DUSP-9) and determine its clinical significance in human cCRCCS.

Methods: The expression of DUSP-9 mRNA was determined in 46 paired samples of cCRCCs and adjacent normal tissues by using real-time qPCR. The expression of the DUSP-9 was determined in 211 samples of cCRCCs and 107 paired samples of adjacent normal tissues by immunohistochemical analysis. Statistical analysis was performed to define the relationship between the expression of DUSP-9 and the clinical features of ccRCC.

Results: The mRNA level of DUSP-9, which was determined by real-time RT-PCR, was found to be significantly lower in tumorous tissues than in the adjacent non-tumorous tissues $(p<0.001)$. An immunohistochemical analysis of 107 paired tissue specimens showed that the DUSP-9 expression was lower in tumorous tissues than in the adjacent non-tumorous tissues ( $p<0.001$ ). Moreover, there was a significant correlation between the DUSP-9 expression in cCRCCs and gender $(p=0.031)$, tumor size $(p=0.001)$, pathologic stage $(p=0.001)$, Fuhrman grade $(p=0.002)$, T stage $(p=0.001)$, $N$ classification $(p=0.012)$, metastasis $(p=0.005)$, and recurrence $(p<0.001)$. Patients with lower DUSP-9 expression had shorter overall survival time than those with higher DUSP-9 expression ( $p<0.001)$. Multivariate analysis indicated that low expression of the DUSP-9 was an independent predictor for poor survival of cCRCC patients.

Conclusion: To our knowledge, this is the first study that determines the relationship between DUSP-9 expression and prognosis in CCRCC. We found that decreased expression of DUSP-9 is associated with poor prognosis in cCRCC. DUSP-9 may represent a novel and useful prognostic marker for cCRCC.
\end{abstract}

\section{Background}

Clear cell renal cell carcinoma is a common urological malignancy worldwide [1]. Although there have been immense improvements in the treatment of ccRCC during recent years, there is a gradual increase in the incidence of this disease. ccRCC initially presents as metastasis in $30 \%$ of patients, and up to $40 \%$ patients undergoing nephrectomy develop local recurrence or

\footnotetext{
* Correspondence: caizhiming2000@yahoo.com.cn; zhoufj@mail.sysu.edu.cn 'Shenzhen Second People's Hospital, The First Affiliated Hospital of

Shenzhen University, Shenzhen 518035, PR China

${ }^{4}$ Department of Urology, Sun Yat-Sen University Cancer Center, Guangzhou 510060, Guangdong, PR China

Full list of author information is available at the end of the article
}

metastatic disease [2]. Although some environmental and genetic factors have been found to be associated with ccRCC, the molecular mechanisms involved in the initiation and progression of ccRCC are still unclear [3].

Dual-specificity phosphatase 9 (DUSP-9) is a member of the dual-specificity protein phosphatase subfamily $[4,5]$. DUSP-9 negatively regulates members of the mitogen-activated protein (MAP) kinase superfamily (e.g., ERK, JNK, p38), which are associated with cellular proliferation and differentiation [6,7]. Massively parallel sequencing studies have revealed the down-regulation of DUSP-9 in ccRCC [8]. However, since there is no published report on this phenomenon, the relationship

\section{Biomed Central}


between the expression of DUSP-9 and clinical significance needs to be clarified. In this study, we aimed to explore the expression of DUSP-9 and its clinical significance in ccRCC.

\section{Methods}

\section{Patients and tissue specimens}

Written informed consent was obtained from all patients, and the study was approved by the institutional review board of Sun Yat-sen University. For real-time RT-PCR analysis, we collected 46 paired samples of ccRCCs and adjacent normal tissues from patients who underwent radical nephrectomy between February 2008 and December 2009. The 46 patients included 40 men and 6 women with a median age of 50 years (range, 3775 years). The fresh tissues were immediately immersed in RNAlater (Qiagen; Germany) after surgical resection, stored at $4^{\circ} \mathrm{C}$ overnight to allow thorough penetration of the tissue, and then frozen at $-80^{\circ} \mathrm{C}$. In addition, we performed an immunohistochemical assay of 211 paraffinembedded samples of ccRCCs and 107 adjacent normal renal tissue samples collected from patients between 1999 through 2007. The characteristics of these 211 patients are listed in Table 1. None of the patients underwent radiotherapy or chemotherapy before surgery. The histological and clinical diagnosis of the tumors in all these patients was performed by the Cancer Center of Sun Yat-sen University. The disease stage of each patient was classified or reclassified according to the 2002 American Joint Committee on Cancer (AJCC) staging system [9].

\section{Real-Time qPCR}

Total RNA was extracted using the TRIzol solution (Invitrogen; Carlsbad, CA) according to the manufacturer's protocol; RNase-free DNase I was used to remove the DNA contamination. M-MLV reverse transcriptase (Fermentas; American) was used according to the manufacturer's recommendations to treat $2 \mu \mathrm{g}$ of the total RNA for synthesizing the ?rst-strand cDNA. The cDNA was then subjected to real-time quantitative PCR for evaluation of the relative mRNA levels of DUSP-9 and GAPDH (as an internal control) with

the corresponding primer pairs ( DUSP-9 sense strand: 5'-TATGCCACGCCCTTTGAG-3', DUSP-9 antisense strand: 5'-CACAGCAGGATGTAGGAGATGA-3'; GAPDH sense strand: 5'-GCTCTCTG CTCCTCCTGTTC-3', GAPDH antisense strand: 5'GACTCCGACCTTCACCTTCC-3'). Gene-speci?c ampli?cation was performed using an Applied Biosystems (ABI 7000) real-time PCR machine with a $20-\mu$ l PCR reaction mixture containing $1 \mu \mathrm{l}$ of cDNA (synthesized as described above), 10- $\mu$ l SYBR Green master mix (Invitrogen; Carlsbad, CA), and $40 \mathrm{nM}$ of each pair of
Table 1 Correlation between DUSP-9 expression and clinical pathologic features of the patients with clear cell renal cell carcinoma

\begin{tabular}{|c|c|c|c|c|c|}
\hline \multirow[t]{2}{*}{ Clinical-pathologic variables } & \multirow[t]{2}{*}{$\mathrm{n}$} & \multicolumn{2}{|c|}{$\begin{array}{l}\text { DUSP-9 } \\
\text { expression }\end{array}$} & \multirow[t]{2}{*}{$\chi^{2}$} & \multirow[t]{2}{*}{$p$} \\
\hline & & Low & High & & \\
\hline All cases & 211 & 117 & 94 & 4.667 & 0.031 \\
\hline Male & 140 & 85 & 55 & & \\
\hline Female & 71 & 32 & 39 & & \\
\hline Age (yrs) & & & & 0.417 & 0.518 \\
\hline$>50$ & 104 & 60 & 44 & & \\
\hline$\leq \mathbf{5 0}$ & 107 & 57 & 50 & & \\
\hline Pathologic stage & & & & 17.112 & 0.001 \\
\hline 1 & 128 & 58 & 70 & & \\
\hline II & 23 & 13 & 10 & & \\
\hline III & 31 & 22 & 9 & & \\
\hline IV & 29 & 24 & 5 & & \\
\hline Fuhrman Grade & & & & 14.492 & 0.002 \\
\hline 1 & 39 & 28 & 11 & & \\
\hline II & 123 & 72 & 51 & & \\
\hline III & 34 & 10 & 24 & & \\
\hline IV & 15 & 7 & 8 & & \\
\hline Tumor size $(\mathrm{cm})$ & & & & 10.906 & 0.001 \\
\hline$\leq 7$ & 136 & 64 & 72 & & \\
\hline$>7$ & 75 & 53 & 22 & & \\
\hline T stage & & & & 14.914 & 0.001 \\
\hline $\mathrm{T}_{1}$ & 135 & 63 & 72 & & \\
\hline $\mathrm{T}_{2}$ & 35 & 21 & 14 & & \\
\hline $\mathrm{T}_{3}, \mathrm{~T}_{4}$ & 41 & 33 & 8 & & \\
\hline $\mathrm{N}$ stage & & & & 6.248 & 0.012 \\
\hline No & 184 & 96 & 88 & & \\
\hline $\mathrm{N}+$ & 27 & 21 & 6 & & \\
\hline Metastasis & & & & 7.708 & 0.005 \\
\hline No & 188 & 98 & 90 & & \\
\hline Yes & 23 & 19 & 4 & & \\
\hline Recurrence & & & & 24.050 & $<0.001$ \\
\hline No & 181 & 88 & 93 & & \\
\hline Yes & 30 & 29 & 1 & & \\
\hline
\end{tabular}

oligonucleotide primers. The ampli?cation conditions were $50^{\circ} \mathrm{C}(2 \mathrm{~min})$ and $95^{\circ} \mathrm{C}(2 \mathrm{~min})$ for 1 cycle and $95^{\circ} \mathrm{C}(15$ sec), $55^{\circ} \mathrm{C}$ (30 sec), and $72^{\circ} \mathrm{C}(40 \mathrm{sec})$ for 40 cycles. Regression curves were calculated for each sample, and the relative amount of mRNA was calculated from the threshold cycles by using the software provided with the instrument (Version 17.0 SPSS Inc.). Relative expression levels of the target genes were normalized to the geometric mean of the internal control gene, GAPDH. The data was analyzed using the comparative threshold cycle $\left(2^{-\Delta \mathrm{CT}}\right)$ method.

\section{Immunohistochemical assay}

An immunohistochemical assay was performed to examine DUSP-9 expression in the 211 ccRCC samples and 
107 paired samples of adjacent normal renal tissue. All procedures were performed using classical protocols. In brief, paraffin-embedded specimens were cut into $5-\mu \mathrm{m}$ sections and baked at $65^{\circ} \mathrm{C}$ for $30 \mathrm{~min}$. The sections were deparaffinized with xylene and rehydrated, submerged into $0.01 \mathrm{M}$ citrate buffer ( $\mathrm{pH}$ 6.0) antigen retrieval buffer, and then microwaved for antigen retrieval. They were then treated with $3 \%$ hydrogen peroxide in methanol to quench the endogenous peroxidase activity, which was followed by incubation with $10 \%$ bovine serum albumin to block nonspecific binding. The DUSP-9 protein was detected by using a mouse monoclonal antibody against DUSP-9 (Abcam; Cambridge, MA, USA). The specimens were incubated overnight at $4{ }^{\circ} \mathrm{C}$ with anti-DUSP- 9 antibody $(1: 250)$. The negative control for immunohistochemical analysis was obtained by replacing the primary antibodies with an antibody diluent. After being washed in phosphate buffered saline (PBS), the sections were treated with MaxVision ${ }^{\mathrm{TM}}$ HRP-Polymer anti-Mouse IHC Kit (Maixin Bio; Fujian, China) at $37^{\circ} \mathrm{C}$ for $15-20 \mathrm{~min}$. The tissue sections were immersed in 3-amino-9-ethyl carbazole, counterstained with Mayer's hematoxylin, dehydrated, and finally mounted in Crystal Mount.

The formalin-fixed, paraffin-embedded sections were reviewed for the degree of immunostaining and scored by 2 independent observers. The proportion of cells expressing DUSP-9 varied from $0 \%$ to $100 \%$, and the intensity of staining varied from weak to strong. The proportion of DUSP-9- expressing tumor cells was scored as follows: 0 , no positive cells; $1,<5 \% ; 2,6 \%-25 \%$; $3,26 \%-50 \% ; 4,51 \%-75 \%$; and $5,>75 \%$ according to Tsuchiya et al. The staining intensity was graded according to the mean optical density [10-12]: 0, no staining; 1 , weak staining (light yellow); 2, moderate staining (yellow brown); and 3, strong staining (brown). Staining index was calculated as the multiplication of staining intensity score and the proportion of DUSP-9-positive tumor cells. We evaluated DUSP-9 expression in benign kidney tissue and malignant lesions on the basis of the staining index values, with scores of $0,1,2,3,4,5,6,8,9,10$, 12 , and 15. The cutoff values for DUSP-9 expression were chosen on the basis of a measure of heterogeneity in overall survival rates, which was calculated using the log-rank test. An optimal cutoff value was identified: a staining index score of $\geq 5$ was considered as high DUSP-9 expression, whereas a staining index score of $\leq 4$ was considered as low DUSP-9 expression.

\section{Statistical analysis}

All statistical analysis was carried out with the SPSS 17.0 statistical software package. In the real-time RT-PCR and immunohistochemical assays, paired-sample $t$ tests were used to analyze the significance of the differences in mRNA and protein expression between ccRCCs and the adjacent normal tissues. The $\chi^{2}$-test for proportion was used to analyze the relationship between DUSP-9 expression and clinical significance. Survival curves were plotted by the Kaplan-Meier method and compared by the log-rank test. We determined that the assumption of proportional hazards was met in all Cox regression models. The significance of various variables for survival was analyzed by the Cox proportional hazards model in multivariate analysis. $\mathrm{p}<0.05$ was considered to be statistically significant.

\section{Results}

\section{Real-time quantitative RT-PCR analysis of DUSP-9} expression

The transcription level of DUSP-9 was determined by quantitative RT-PCR assays of 46 ccRCC tumor samples and the paired adjacent normal tissue samples. In 45 tumor samples, the mRNA level of DUSP-9 was significantly lower than that in the adjacent normal tissue sample $(\mathrm{p}<0.001$, paired-sample $t$ tests, Figure 1$)$.

Immunohistochemical analysis of the expression of DUSP9 protein in 107 paraffin-embedded cCRCC samples (T) and the paired adjacent normal renal tissue $(\mathrm{N})$

Expression and subcellular localization of protein were determined by immunohistochemical analysis in 107 paraffin-embedded ccRCC tissues and 107 paired specimens of adjacent normal tissues (Figure 2). In normal renal tissue, specific DUSP-9 was localized mainly in the cytoplasm of renal cells in the form of yellow-brown granules (Figure 3A). The DUSP-9 protein expression in the 102 tumor tissue samples was lower than that in the adjacent normal tissue samples ( $\mathrm{p}<0.001$, paired-sample $t$ test, Figure 3B).

\section{Immunohistochemical analysis of DUSP-9 expression in 211 cCRCC samples $(T)$ and its relationship with the clinical features}

To further investigate the effect and the prognostic value of DUSP-9, immunohistochemical analysis was performed to assess the expression of DUSP-9 in 211 ccRCC tissue blocks. Overall, 117 of the 211 tumor samples showed low expression of DUSP-9(score $\leq 4)$, whereas 94 samples showed high expression (score $\geq 5$ ). The correlation between the expression of DUSP-9 and various clinicopathological parameters are listed in Table 1. Intense expression of DUSP-9 in ccRCC samples was correlated with gender $(\mathrm{p}=0.031)$, pathologic stage $(p=0.001)$, Fuhrman grade $(p=0.002)$, tumor size $(\mathrm{p}=0.001), \mathrm{T}$ stage $(\mathrm{p}=0.001), \mathrm{N}$ classification $(\mathrm{p}$ $=0.012)$, metastasis $(\mathrm{p}=0.005)$, and recurrence $(\mathrm{p}<$ $0.001)$, but it was not correlated with age. Low expression of DUSP-9 was noted in $46.7 \%, 60.0 \%$, and $80.5 \%$ 


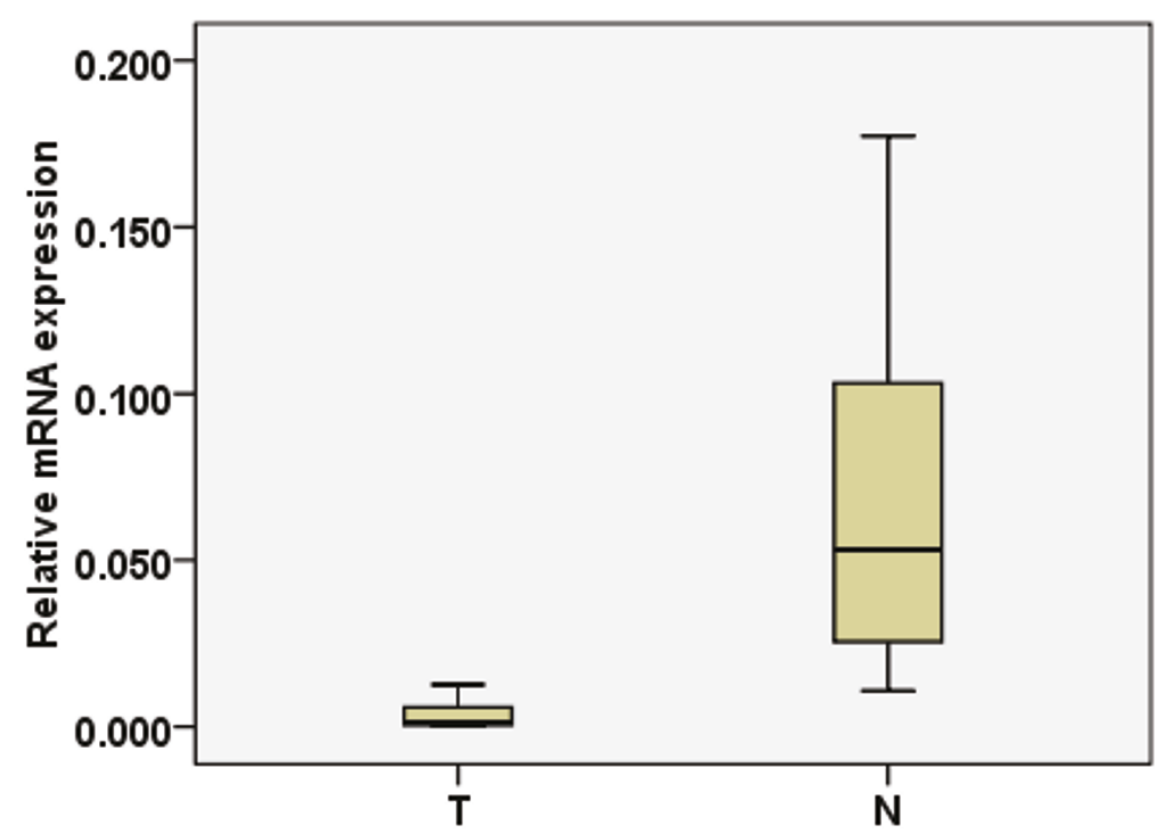

Figure 1 Real-time quantitative RT-PCR analysis of DUSP-9 expression. The relative expression of DUSP-9 mRNA in RCC tumor tissue samples was lower than that in the paired adjacent normal $(N)$ tissue samples $(n=46, P<0.001)$. The bottom and the top of the box represent the $25^{\text {th }}$ and the $75^{\text {th }}$ percentile, respectively, and the band near the middle of the box is the $50^{\text {th }}$ percentile (the median). The ends of the whiskers represent the $2.5^{\text {th }}$ percentile and the $97.5^{\text {th }}$ percentile.

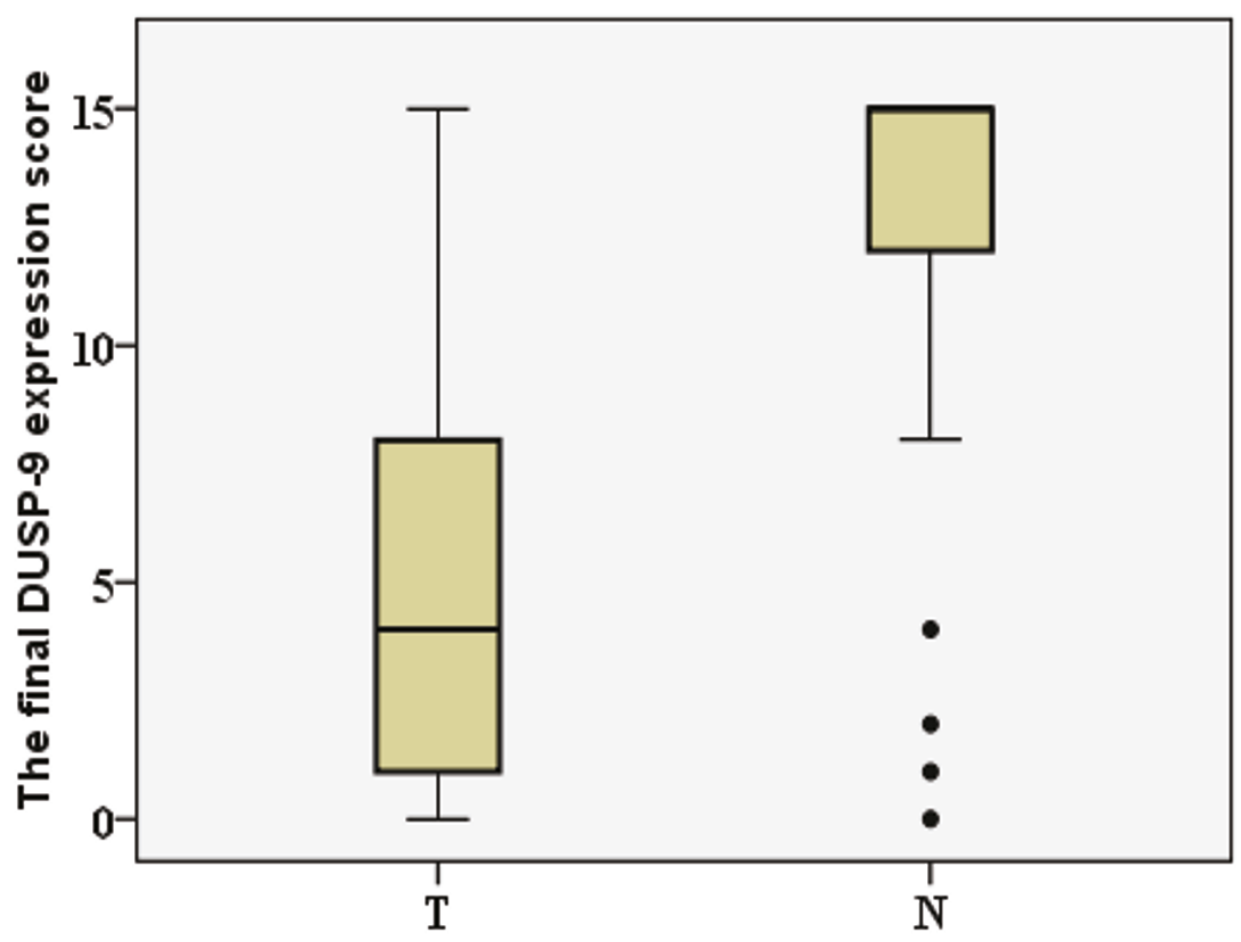

Figure 2 Decreased protein expression of DUSP-9 in ccRCC. The relative protein expression of DUSP-9 in ccRCC tumor (T) tissue samples was lower than that in the paired adjacent normal $(N)$ tissue samples $(n=107, P<0.001)$. The bottom and top of the box are the lower and upper quartiles, and the band near the middle of the box is the median. The ends of the whiskers represent the $2.5^{\text {th }}$ percentile and the $97.5^{\text {th }}$ percentile. Four black spots represent the special value outliers. 


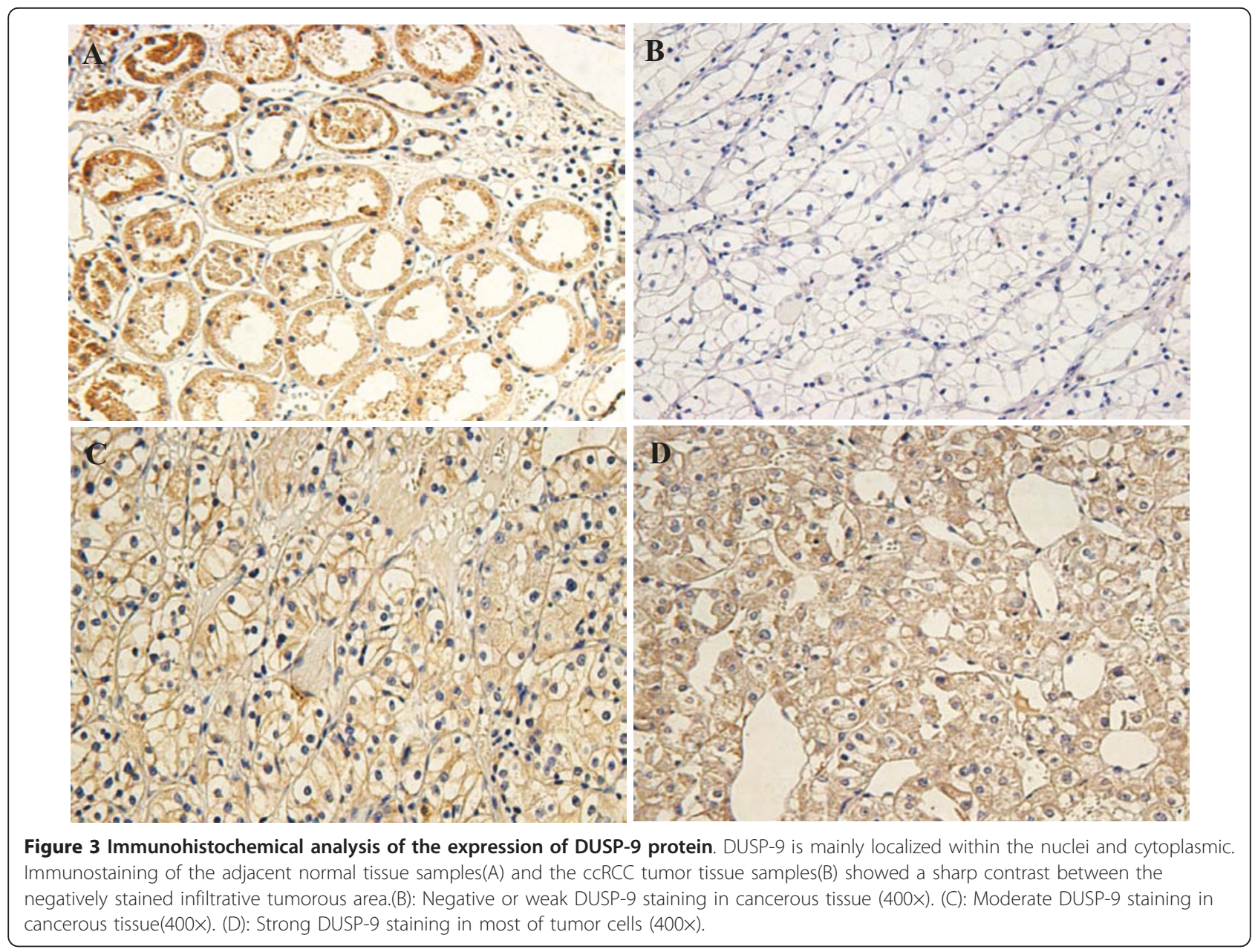

of $\mathrm{T} 1, \mathrm{~T} 2$, and $\mathrm{T} 3 / 4$ stage ccRCCs respectively ( $\mathrm{p}=$ $0.001, \chi 2$ test). Low expression of DUSP-9 was not observed in $47.1 \%$ and $70.7 \%$ of ccRCCs with size $\leq 7 \mathrm{~cm}$ and $>7 \mathrm{~cm}$ respectively $(\mathrm{p}=0.001, \chi 2$ test). Low expression of DUSP-9 was seen in $52.2 \%$ and $77.8 \%$ of N0 and $\mathrm{N} 1 / 2$ stage ccRCCs respectively $(\mathrm{p}=0.012, \chi 2$ test). Low expression of DUSP-9 was seen in $52.1 \%$ and $82.6 \%$ of ccRCCs with or without metastasis respectively ( $\mathrm{p}=$ $0.005, \chi 2$ test). Low expression of DUSP-9 protein was seen in $48.6 \%$ and $96.6 \%$ of ccRCCs with or without recurrence respectively $\left(\mathrm{p}<0.001, \chi^{2}\right.$ test $)$.

\section{Survival analysis}

Kaplan-Meier analysis and the log-rank test were used to calculate the effect of the DUSP-9 expression on survival. The 5-year survival in the group of patients with high DUSP-9 expression was $97 \%$, but it was $62.1 \%$ in the group of patients with low DUSP-9 expression (Figure $4 \mathrm{~A}$ ). The log-rank test showed that survival rates were significantly different between these 2 groups ( $\mathrm{p}<$ 0.001). Furthermore, the relationship of DUSP-9 expression with prognosis was determined in 211 patients, which were divided into 3 subgroups depending on the pathologic stage. Patients with tumors exhibiting high DUSP-9 expression had significantly longer overall survival than those with low expression of DUSP-9 either in the stage I plus II subgroup $(\mathrm{n}=151 ; \log$-rank, $\mathrm{p}=$ 0.023 ; Figure $4 B)$, the stage III sub group $(n=31$; logrank, $\mathrm{p}=0.036$; Figure $4 \mathrm{C}$ ), or the stage IV subgroup ( = 29; log-rank, $\mathrm{p}=0.038$; Figure 4D). Patients with tumors high DUSP-9 expression had significantly longer overall survival than those with low expression of DUSP-9 either in the Fuhrman grade I subgroup $(\mathrm{n}=39$; $\log$-rank, $\mathrm{p}=0.005$; Additional file 1 Figure S1A), II subgroup $(\mathrm{n}=123$; log-rank, $\mathrm{p}<0.0001$; Additional file 1 Figure S1B), the stage III sub group $(\mathrm{n}=34$; log-rank, $\mathrm{p}<0.001$; Additional file 1 Figure S1C), or the stage IV subgroup $(\mathrm{n}=15 ; \log$-rank, $\mathrm{p}=0.019$; Additional file 1 Figure S1D).

Univariate Cox regression analysis showed that tumor size, $\mathrm{T}$ stage, $\mathrm{N}$ stage, metastasis, Fuhrman grade and DUSP-9 expression were significantly associated with overall survival (Table 2). Furthermore, multivariate Cox regression analysis revealed that only DUSP-9 


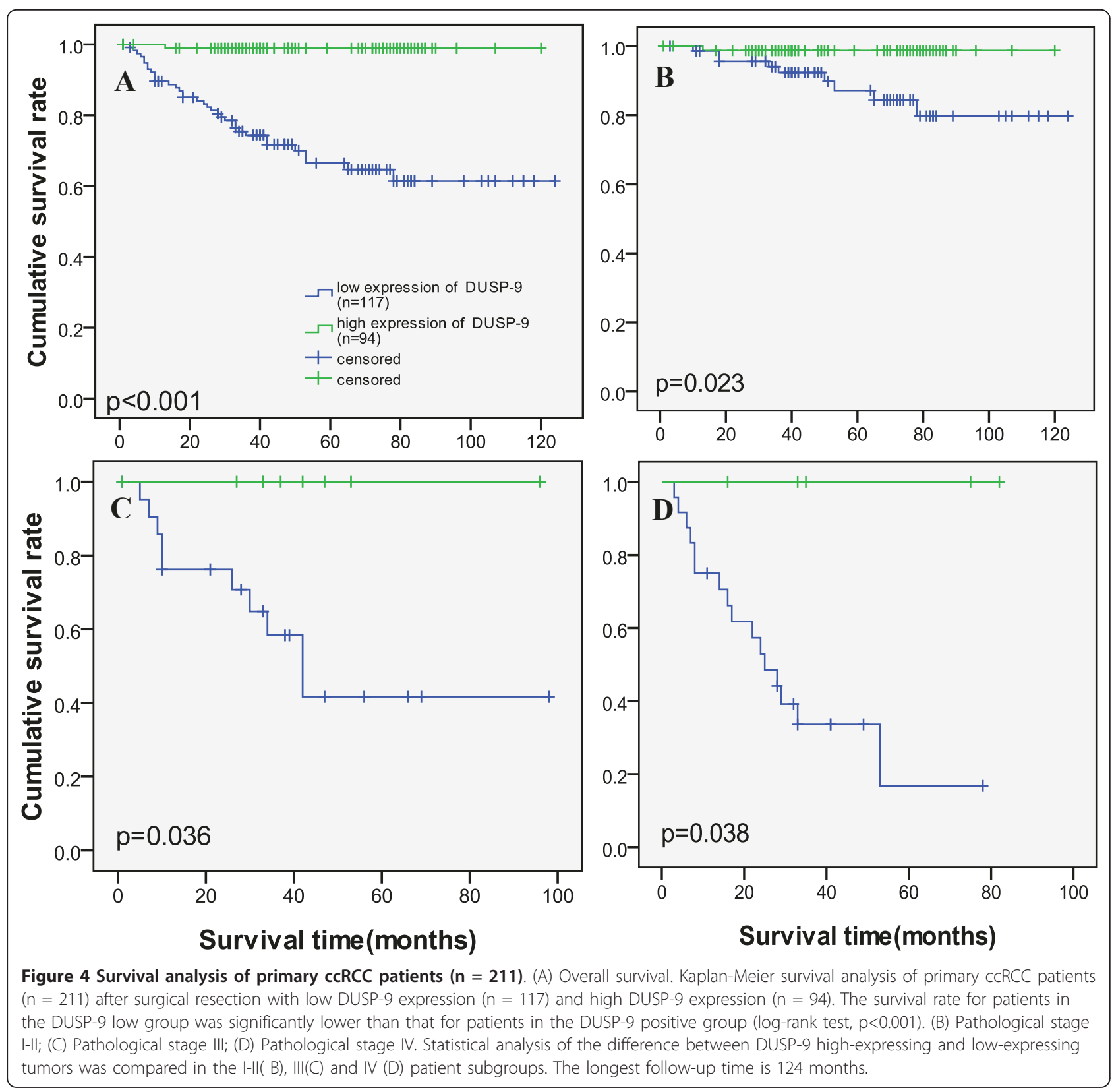

expression and Fuhrman grade were independent predictors for the overall survival of ccRCC patients $(\mathrm{p}=$ 0.005 , respectively; Table 2 ), whereas the others factors were not independently related to the survival of ccRCC patients.

\section{Discussion}

Clear cell renal cell carcinoma accounts for about $2 \%$ of all cases of cancers, with an annual increase of 1.5-5.9\% worldwide $[13,14]$. The initial treatment is usually a radical or partial nephrectomy, which remains the mainstay of curative treatment [15]. Unfortunately, ccRCC is resistant to radiation therapy and chemotherapy, but some tumors respond to molecular-targeted therapy. Therefore, identification of specific molecular biomarkers of ccRCC is an essential prerequisite. Although the numerous molecular markers, such as p53, vascular endothelial growth factor (VEGF), hypoxia inducible factor, Ki67 (proliferation), have been investigated as prognostic variables in ccRCC, the molecular mechanisms of the initiation and progression of ccRCC still remain unclear [16,17]. Massively parallel sequencing analysis showed that DUSP-9 is downregulated in ccRCC [9].

DUSP-9 is a member of the dual-specificity protein phosphatase subfamily and is expressed only in the placenta, kidney, and during the fetal life. Moreover, 
Table 2 Cox Regression analysis of the overall survival rates associated with different prognostic variables in patients with ccRCC

\begin{tabular}{ccccc}
\hline Variables & \multicolumn{2}{c}{ Univariate analysis } & \multicolumn{2}{c}{ Multivariate analysis } \\
\cline { 2 - 5 } & $\begin{array}{c}\text { Hazard ratios } \\
\text { (95\% confidence interval) }\end{array}$ & $\mathrm{p}$ & $\begin{array}{c}\text { Hazard ratios } \\
(95 \% \text { confidence interval) }\end{array}$ & $\mathrm{p}$ \\
\hline Tumor size & $(3.339-15.178)$ & $<0.001$ & $(0.088-3.118)$ & 0.478 \\
T stage & $(2.177-4.177)$ & $<0.001$ & $(0.629-5.052)$ & 0.276 \\
N stage & $(1.389-4.379)$ & $=0.002$ & $(0.438-3.119)$ & 0.755 \\
Metastasis & $(2.705-11.837)$ & $<0.001$ & $(0.100-6.355)$ & 0.832 \\
Fuhrman Grade & $(35.53-630.43)$ & $<0.001$ & $(27.23-569.2)$ & $<0.001$ \\
DUSP-9 & $(0.004-0.229)$ & 0.001 & $(0.088-3.118)$ & 0.008 \\
\hline
\end{tabular}

DUSP-9 is known to be associated with squamous cell carcinoma (SCC) and can independently induce SCC [5]. DUSP-9 inactivates the target kinases of squamous carcinoma cells by dephosphorylating both the phosphoserine/threonine and phosphotyrosine residues. DUSP-9 negatively regulates members of the mitogen-activated protein (MAP) kinase superfamily (MAPK, p38, SAPK), which are associated with cellular proliferation and differentiation [18-20]. Molly Kulesz-Martin et al. found that DUSP-9 reconstitution resulted in G2-M-associated cell death and microtubule disruption. Loss of DUSP-9 was associated with SCC, and it independently induced SCCs relative to benign tumors in mouse skin. Reconstitution of DUSP-9 expression in malignant tumor cells induces cell death and tumor suppression [6,21,22].

However, to our knowledge, the key feature of this study is that this is the first study to report the clinical significance of DUSP-9 in ccRCC. This is also the first study aimed at evaluating the possibility of using DUSP9 as a clinically potential indicator for disease progression, as well as a prognostic marker for patient survival in tumors.

In this study, we showed that DUSP-9 mRNA and protein expression were significantly different between the ccRCC and the adjacent normal renal tissue samples. Furthermore, immunohistochemical analysis showed that DUSP-9 expression was moderate to low in ccRCCs, while it was high in the adjacent normal tissues. Accordingly, we found that DUSP-9 expression was reduced in a large number of human clinical ccRCC samples. The decreased expression of DUSP-9 was correlated with gender, pathologic stage, Fuhrman grade, tumor size, recurrence, TNM stage, and prognosis. Patients with lower DUSP-9 expression had shorter survival time, and those with higher DUSP-9 expression had a longer survival time. In addition, the relationship of DUSP-9 expression with prognosis was determined in the patients, which were divided into 3 subgroups depending on the pathologic stage. We found that DUSP-9 could be a valuable prognostic marker for ccRCC patients at all disease stages. Consistent with previous reports of other cancers, low-expression of DUSP-9 indicated poor prognosis for patients with ccRCC.

DUSP-9 expression is correlated with low Fuhrman grade. This result did not match with the other correlations. However, in the survival analysis, we found that patients with tumors high DUSP-9 expression had significantly longer overall survival than those with low expression of DUSP-9 either in the Fuhrman grade I subgroup, II subgroup, the stage III sub group, or the stage IV subgroup. We observed that there are more cases with low Fuhrman grade. In addition, this was a single hospitalbased, retrospective study. In addition to this observation, we have, in particular, found that DUSP-9 expression is correlated with low Fuhrman grade.

The TNM stage of ccRCC and Fuhrman grade are closely related to its prognosis [23-25]. In our study, the results of univariate Cox regression analysis showed that tumor size, $\mathrm{T}$ stage, $\mathrm{N}$ stage, metastasis, Fuhrman grade and DUSP-9 expression were significantly associated with overall survival. Furthermore, multivariate Cox regression analysis revealed that only DUSP-9 expression and Fuhrman grade were independent predictors for the overall survival of ccRCC patients. Thus, our findings indicate that the DUSP-9 expression level has a significant correlation with clinicopathological features and is a potential prognostic marker for ccRCC.

Our study was a single hospital-based, retrospective study. It should be pointed out that unmeasured differences may exist and may distort the study results. A multicenter or community-based prospective study with more extensive collection of potential confounders is required. In addition, the correlation of DUSP-9 with the abovementioned molecular markers needs to be investigated further. Apparently, more studies are required to explore the relationship between the DUSP-9 gene and other genes such as p38 that may be associated with ccRCC.

\section{Conclusion}

In summary, we demonstrated the down-regulation of DUSP-9 in ccRCC and its correlation with poor 
prognosis by using a large number of clinical samples. Our results indicate the role of DUSP-9 as a prognostic factor and a potential tumor suppressor in primary ccRCC. Measurement of DUSP-9 expression in primary ccRCC can help stratify the patients for prognosis. Furthermore, DUSP-9 may be a new potential therapeutic target for ccRCC in the future.

\section{Additional material}

Additional file 1: Additional file 1, Figure S1. Survival analysis of difference Fuhrman grade.

\section{Acknowledgements}

This project was supported by the Promotion Program for Shenzhen Key Laboratory, Shenzhen, China (CXB200903090055A andCXB201005250016A to Z.M.-C.) and the Science Foundation of Shenzhen, China (CXC201005260001A).

\section{Author details}

'Shenzhen Second People's Hospital, The First Affiliated Hospital of Shenzhen University, Shenzhen 518035, PR China. ${ }^{2}$ Institute of Urology, Shenzhen PKU-HKUST Medical Center, Shenzhen 518036, Guangdong, PR China. ${ }^{3}$ Anhui Medical University, Hefei 230022, An Hui, PR China. ${ }^{4}$ Department of Urology, Sun Yat-Sen University Cancer Center, Guangzhou 510060, Guangdong, PR China.

\section{Authors' contributions}

SW, YW, LS, ZLZ were responsible for data collection and analysis, experiment job, interpretation of the results, and writing the manuscript. $\mathrm{HH}$ $Z K Q, Y T G, X X L, A F T, Z W L$ and $M Z J$ were responsible for conducting the data analysis, reviewing and scoring the degree of immunostaining of sections in cooperation with LS. FJZ and ZMC were responsible for experimental design, analysis and interpretation. All authors have read and approved the final manuscript.

\section{Competing interests}

The authors declare that they have no competing interests.

Received: 20 March 2011 Accepted: 26 September 2011 Published: 26 September 2011

\section{References}

1. McLaughlin JK, Lipworth L, Tarone RE: Epidemiologic aspects of renal cell carcinoma. Semin Oncol 2006, 33:527-523.

2. Motzer RJ, Bander NH, Nanus DM: Renal-cell carcinoma. N Engl J Med 1996, 335:865-875.

3. Janzen NK, Kim HL, Figlin RA, Belldegrun AS: Surveillance after radical or partial nephrectomy for localized renal cell carcinoma and management of recurrent disease. Urol Clin North Am 2003, 30:843-852.

4. Christie GR, Williams DJ, Macisaac F, Dickinson RJ, Rosewell I, Keyse SM: The dual-specificity protein phosphatase DUSP9/MKP-4 is essential for placental function but is not required for normal embryonic development. Mol Cell Biol 2005, 25:832-833.

5. Lewis TS, Shapiro PS, Ahn NG: Signal transduction through MAP kinase cascades. Adv Cancer Res 1998, 74:49-139.

6. Liu Y, Lagowski J, Sundholm A, Sundberg A, Kulesz-Martin M: Microtubule disruption and tumor suppression by mitogen-activated protein kinase phosphatase 4. Cancer Res 2007, 67:10711-10719.

7. Mansour SJ, Matten WT, Hermann AS, Candia JM, Rong S, Fukasawa K, Vande Woude GF, Ahn NG: Transformation of mammalian cells by constitutively active MAP kinase kinase. Science 1994, 265:966-970

8. Zhou L, Chen J, Li Z, Li X, Hu X, Huang Y, Zhao X, Liang C, Wang Y, Sun L, Shi M, Xu X, Shen F, Chen M, Han Z, Peng Z, Zhai Q, Chen J, Zhang Z, Yang R, Ye J, Guan Z, Yang H, Gui Y, Wang J, Cai Z, Zhang X: Integrated
Profiling of MicroRNAs and mRNAs: MicroRNAs Located on Xq27.3 Associate with Clear Cell Renal Cell Carcinoma. PLOS ONE 2010, 5:e15224.

9. Greene FL, Balch CM, Fleming ID, Fritz A, Haller DG, Morrom M, David L, AJCC: Cancer Staging Manual. New York: AJCC Springer-Verlag; 6 2002, 335-340.

10. Tsuchiya A, Sakamoto M, Yasuda J, Chuma M, Ohta T, Ohki M, Yasugi T, Taketani $Y$, Hirohashi S: Expression profiling in ovarian clear cell carcinoma: identification of hepatocyte nuclear factor- 1 beta as a molecular marker and a possible molecular target for therapy of ovarian clear cell carcinoma. Am J Pathol 2003, 163:2503-2512.

11. Saussez S, Cucu DR, Decaestecker C, Chevalier D, Kaltner H, André S, Wacreniez A, Toubeau G, Camby I, Gabius HJ, Kiss R: Galectin 7 (p53induced gene 1): a new prognostic predictor of recurrence and survival in stage IV hypopharyngeal cancer. Ann Surg Oncol 2006, 13:999-1009.

12. Bao S, Ouyang G, Bai X, Huang Z, Ma C, Liu M, Shao R, Anderson RM, Rich JN, Wang XF: Periostin potently promotes metastatic growth of colon cancer by augmenting cell survival via the Akt/PKB pathway. Cancer Cell 2004, 5:329-339.

13. Chow WH, Dong LM, Devesa SS: Epidemiology and risk factors for kidney cancer. Nat Rev Urol 2010, 7:245-257.

14. McCredie M: Bladder and kidney cancers. Cancer Surv 1994, 19:343-368.

15. Ljungberg B, Cowan NC, Hanbury DC, Hora M, Kuczyk MA, Merseburger AS, Patard JJ, Mulders PF, Sinescu IC: European Association of Urology Guideline Group:EAU guidelines on renal cell carcinoma: the 2010 update. Eur Urol 2010, 58:398-406.

16. Sabatino M, Kim-Schulze S, Panelli MC, Stroncek D, Wang E, Taback B, Kim DW, Deraffele G, Pos Z, Marincola FM, Kaufman HL: Serum vascular endothelial growth factor and fibronectin predict clinical response to high-dose interleukin-2 therapy. J Clin Oncol 2009, 27:2645-2652.

17. Banks RE, Craven RA, Harnden P, Madaan S, Joyce A, Selby PJ: Key clinical issues in renal cancer: a challenge for proteomics. World J Urol 2007, 25:537-556.

18. Brondello JM, Brunet A, Pouyssegur J, McKenzie FR: The dual specificity mitogen-activated protein kinase phosphatase- 1 and -2 are induced by the p42/p44MAPK cascade. J Biol Chem 1997, 272:1368-1376.

19. Keyse SM: Protein phosphatases and the regulation of mitogen-activated protein kinase signalling. Curr Opin Cell Biol 2000, 12:186-192.

20. Sun H, Tonks NK, Bar-Sagi D: Inhibition of Ras induced DNA synthesis by expression of the phosphatase MKP-1. Science 1994, 266:285.

21. Liao Q, Guo J, Kleeff J, Zimmermann A, Büchler MW, Korc M, Friess H: Down-regulation of the dual-specificity phosphatase MKP-1 suppresses tumor-igenicity of pancreatic cancer cells. Gastroenterology 2003, 124:1830-1845.

22. Solit DB, Garraway LA, Pratilas CA, Sawai A, Getz G, Basso A, Ye Q, Lobo JM, She Y, Osman I, Golub TR, Sebolt-Leopold J, Sellers WR, Rosen N: BRAF mutation predicts sensitivity to MEK inhibition. Nature 2006, 439:358-362.

23. Zhang Z, Li J, Zheng H, Yu C, Chen J, Liu Z, Li M, Zeng M, Zhou F, Song L: Expression and cytoplasmic localization of SAM68 is a significant and independent prognostic marker for renal cell carcinoma. Cancer Epidemiol Biomarkers Prev 2009, 18:2685-2693.

24. Buchner A, Castro M, Hennig A, Popp T, Assmann G, Stief CG, Zimmermann W: Down-regulation of HNF-1B in renal cell carcinoma is associated with tumor progression and poor prognosis. Urology 2010, 76 : e6-11.

25. Levi F, Ferlay J, Galeone C, Lucchini F, Negri E, Boyle P, La Vecchia C: The changing pattern of kidney cancer incidence and mortality in Europe. BJU Int 2008, 101:949-958.

\section{Pre-publication history}

The pre-publication history for this paper can be accessed here: http://www.biomedcentral.com/1471-2407/11/413/prepub

doi:10.1186/1471-2407-11-413

Cite this article as: Wu et al:: Decreased expression of dual-specificity phosphatase 9 is associated with poor prognosis in clear cell renal cell carcinoma. BMC Cancer 2011 11:413. 